RESEARCH ARTICLE

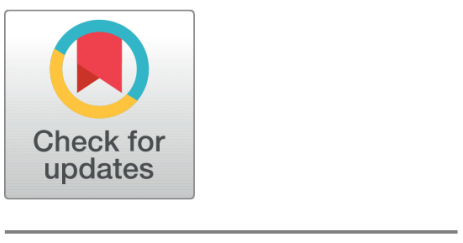

OPEN ACCESS

Received: 28-04-2020

Accepted: 22-07-2020

Published: 28-08-2020

Editor: Dr. Natarajan Gajendran

Citation: Kiran Kumar G, Malathi Rani D, Guru Mohan Rao E (2020) Fast palmprint retrieval using speed up robust features. Indian Journal of Science and Technology 13(31): 3204-3212. https://doi.org/ 10.17485/IJST/v13i31.404

* Corresponding author. ganipalli.kiran@gmail.com

Funding: None

Competing Interests: None

Copyright: (c) 2020 Kiran Kumar et al. This is an open access article distributed under the terms of the Creative Commons Attribution License, which permits unrestricted use, distribution, and reproduction in any medium, provided the original author and source are credited.

Published By Indian Society for Education and Environment (iSee)

ISSN

Print: 0974-6846

Electronic: 0974-5645

\section{Fast palmprint retrieval using speed up robust features}

\author{
G Kiran Kumar ${ }^{1 *}$, D Malathi Rani², E Guru Mohan Rao ${ }^{3}$ \\ 1 Professor, Department of Information Technology, MLR Institute of Technology, \\ Hyderabad, 500043, India \\ 2 Assistant Professor, ECE Department, Marri Laxman Reddy Institute of Technology and \\ Management, Hyderabad, 500043, India \\ 3 Assistant Professor, Department of Information Technology, MLR Institute of Technology, \\ Hyderabad, 500043, India
}

\section{Abstract}

Background/Objective: Biometric usage is increasing in exponential series in all organisations for multiple purposes like employee attendance, Aadhaar based authentication and secure login using finger print etc. This biometric process should be as quick as possible without making much delay to retrieve the respective finger print. So an efficient quick retrieval procedure is required, in this regards a fast retrieval method for palm prints is proposed in this article. Method: This method uses Speed up Robust Features (SURF) and an efficient look up table for fast retrieval of palm prints. A key is computed for each palmprint by matching with a pre-selected palmprint called representative. This key is used, to place the palmprint into the look up table like traditional database record. To identify a query palmprint, key is computed and selects a set of palm prints from the look up table which are having similar key as possible matches. Findings: This proposed solution is experimented with multiple representative images to check the improved performance. As an outcome we could achieve better hit rate by comparing with existing system. Novelty: This proposed method makes the new palm prints dynamically without disturbing the current records in the system. The entire solution is experimented on benchmark PolyU palmprint database of 7,753 images and significant performance is shown in results. This proposed solution shows better results with respect to hit rate and miss rate.

Keywords: Palmprint; index key; SURF; similarity score; representative

\section{Introduction}

Nowadays security is an important issue in every sector including government, business, etc. Therefore, the use of biometric systems has been increased enormously in every field. However, many of these biometric frameworks has to manage huge databases and its size is expanding at a quick pace. For example, India's national ID program known as Unique Identification Authority of India (UIDAI) has a database of more than 700 million individuals. It may reach 1.25 billion individuals in last couple 
of years.

Generally, recognition of a person in these biometric applications is done by comparing the person's biometric sample with all registered samples in the dataset. This linear matching process increases the search time of the system. Hence, an efficient indexing procedure or method is required and those methods can enable searches over a large database in less time and without compromising recognition accuracy. The indexing method should be designed such that, to identify a query, it has to immediately fetch a small group of candidates which are most alike from the database. Then, as a replacement of comparing the query with the whole database, only the samples in the candidate set are compared which increases the query speed and reduces the response time of the system ${ }^{(1)}$.

An indexing technique generates an index key to all registered image in the database. How-ever, the below issues to be viewed before to construct index keys: (1) As biometric image accession and refining is liable to noise, the biometric sample acquired from a user in the process of spotting may be non - identical from the sample which was utilized to create the framework during enrolment. Which in turn results in dissimilar index keys for the same user and the recognition process becomes difficult. (2) Traditionally, database records are arranged in sorting order for efficient retrieval based on either alphabetically or numerically. But, we cannot set out the biometric pictures similar to traditional records as they won't have any regular sorting sequence to index ${ }^{(2)}$. Thus, traditional ways are not applicable for biometric images.

Remaining manuscript is arranged as follows. The current biometric indexing techniques will be explained in Section 2 . Section 3 explains the proposed indexing technique. Experimental outputs are provided in Section 4. Section 5 concludes the paper.

\section{Related work}

The current biometric indexing techniques can be vastly divided into: i) point, ii) triplet or quadruplet of points, or iii) match score based approaches. In point based approaches, authors taken the important factors of the biometric samples and utilized geometric hashing or its variants to do indexing ${ }^{(3,4)}$. In triplet based indexing approaches, author first compute a set of triangles from the extracted feature points of the biometric samples ${ }^{(5-9)}$. The computed triplets are assigned to a hash table utilizing their geometric features like angles, lengths of the sides, orientation, type and many more. Iloanusi, computed the quadruplets from the key feature points and used the geometric properties calculated from them for indexing purpose ${ }^{(10)}$. However, the drawback of all the methods de-scribed above is that, the features extracted for different samples are not equal in number which makes the system unreliable.

Further, there are some logics apply on similarity scores for indexing which deal with fixed length keys ${ }^{(1-15)}$. However, most of these methods fail to register the new users dynamically and used sequential search over the index space to identify a query. This paper addresses the above problems and investigates an accurate technique to index palmprints using SURF features ${ }^{(16-18)}$ . A look up table is created to index the biometric samples like traditional database records. This allows the identification approach to avoid the linear search during identification of a query and performs a quick search. Further, this approach creates entry to the new users dynamically without disturbing the existing system.

In this paper ${ }^{(19)}$, they mainly worked on 2-D palm print images. To recognize the palm print they proposed a method where touch of palm doesn't require. Convolutional Neural Network $(\mathrm{CNN})$ approach is used to extract features of the palm that are highly discriminated. Earlier methods are based on supervised learning for which class labels are required. But in this approach unsupervised procedures are adopted through Gabor responses and Principle Component Analysis (PCA). Though there are many approaches such as coding - based approaches, Local Texture Descriptor based approaches and Deep Learning based approaches, in this paper touch less palm prints are identified by PalmNet and it is a novel CNN based method ${ }^{(20)}$.

In this paper ${ }^{(21)}$, the authors state that, basically there are two types of palmprint recognition: First is online palm print identification and second is latent palmprint identification. The first one will happen with a digital camera where the user of palm print will cooperate to take palm print. Second is palm print collected from crimes scenes, where user obviously does not cooperate. In second case usually criminals may hide their faces, but we can use their palm print from the whole image to identify the person. In this paper, a new palm print database is established and applied a new deep learning method.

In this paper ${ }^{(22)}$, the authors proposed a method to retrieve the palmprint using Deep Convolutional Neural Network (DCNN). In this method, large database set has been taken for experiment their proposed idea. Few research articles summarised ${ }^{(23)}{ }^{(24)}{ }^{(25)}$ all the palmprint retrieval methods.

\section{Proposed indexing technique}

This technique relies mainly on similarity scores between the palmprints. The similarity score between 2 palmprints is calculated by comparing their SURF features ${ }^{(4,26-31)}$ in Euclidean space. 
Table 1. Look up table for the images

\begin{tabular}{ll}
\hline Score with $\mathbf{r}($ key $)$ & List of images \\
\hline 0 & $\mathrm{I}_{I D}$ LIST \\
1 & $\mathrm{I}_{I D}$ LIST \\
$\cdot$ & $\cdot$ \\
$\cdot$ & $\cdot$ \\
$\mathrm{S}-1$ & $\mathrm{I}_{I D}$ LIST \\
$\mathrm{S}$ & $\mathrm{I}_{I D}$ LIST \\
$\mathrm{S}+1$ & $\mathrm{I}_{I D}$ LIST \\
$\cdot$ & $\cdot$ \\
$\cdot$ & $\cdot$ \\
100 & $\mathrm{I}_{I D}$ LIST \\
\hline
\end{tabular}

The concept behind using similarity scores is that, generally palmprints that belongs to same user will be having similar features and their similarity scores with a third palmprint (say representative) are also equal (Equation 3.1).

$$
\text { if }(A=B)) \Rightarrow[S(A, r)=S(B, r)]
$$

where $A$ and $B$ be are two palmprints of a user, $r$ is a representative palmprint, $S(A, r)$ and $S(B, r)$ are the similarity scores of palmprint $A$ and palmprint $B$ with $r$ respectively. This motivates us to use the similarity score of each palmprint as its index key and we use this key to make entry into a look up table ( Figure 1 ). Let A be a palmprint and s is its key, then A is registered to $\mathrm{s}^{\text {th }}$ location of A. It can be observed from Figure 1 that, the palmprint IDs are organized in sorted order based on their keys like traditional database records. During identification, first we compute the key of the query by matching it with $r$. Next, using this key we retrieve the palmprints that are having similar key in the look up table ( Figure 1). This is given in Equation 3.2:

$$
C+\left\{I_{i d} \text { List | }\left[S(q, r)=S\left(I_{i d} \text { List }, r\right)\right]\right\}
$$

where $\mathrm{C}$ is the set of candidates retrieved as similar matches, $\mathrm{q}$ is the query palmprint and $\mathrm{I}_{i d}$ List is the palmprint IDs in the look up table, those are similar to q.

The list of similar palmprints for a query can be retrieved in $\mathrm{O}(1)$ time, as the palmprints in the look up table are already arranged in sorted manner based on their keys. However, this retrieved list contains some palmprints that are dissimilar (false matches) to q but have similar key. For example, alphabets $\mathrm{X}$ and $\mathrm{Z}$ have same distance (i.e., score) to $\mathrm{Y}$ in the sequence even though $\mathrm{X}$ and $\mathrm{Z}$ are different. These false matches can be filtered out by choosing more number of representative palmprints. For each representative, a look up table is created. The user palmprints are made and entry into each table according to their score (key) with the corresponding representative. During identification of a query palmprint, each look up table produces a various set of candidate palmprints as similar matches. We select the palmprints which are repeated in most of the sets as final candidates for the query.

Note that, this process filter out the false matches and increases the identification accuracy of the system.

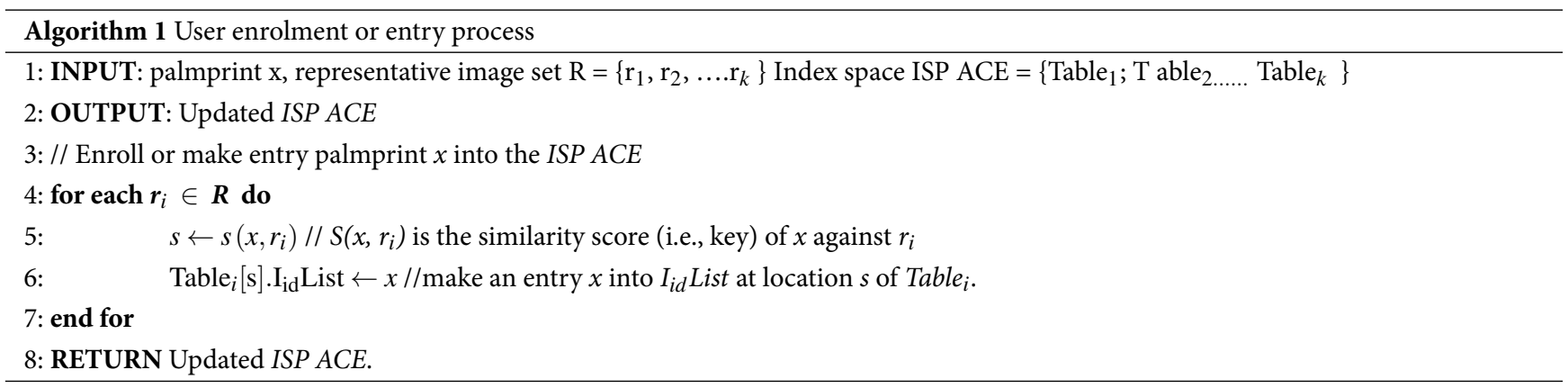

\subsection{User enrolment or make an entry}

Let $\mathrm{R}=\left\{\mathrm{r}_{1}, \mathrm{r}_{2}, \ldots . . \mathrm{r}_{k}\right\}$ be the representative image set. We create a separate look up table Table $e_{i}$ for each $\mathrm{r}_{i}$, where $1 \leq \mathrm{i} \leq \mathrm{k}$. The palmprints are enrolled into each Table $i$ based on their key (i.e., score) with the corresponding $\mathrm{r}_{i}$. The process of enrolling a 
palmprint is described in Algorithm 1. Let a be a user palmprint to make an entry and let $s=S\left(a, r_{1}\right)$ be the key of it with $r_{1}$. We access Table $_{1}$ and registered palmprint a into location s. We repeat this process with other representative palmprints and register a into corresponding look up tables.

\subsection{Retrieval of similar palmprints for a query}

In this section, the proposed is discussed i.e retrieval technique that identifies a set of potential (i:e:; similar) candidates from its database for a query palmprint. To identify the similar candidates for a query palmprint $\mathrm{q}$, it is matched against each representative image and computes the corresponding index key. Let $\mathrm{x}\left(=\mathrm{Key}_{i}\right)$ be the index key of the query against $\mathrm{r}_{i}$, we access $\mathrm{x}^{\text {th }}$ location in $\mathrm{Table}_{i}$ and retrieve the $\mathrm{I}_{i d}$ List from there. We repeat this process with all keys [Key $]^{\mathrm{k}}{ }_{i=1}$ of the query palmprint and retrieve the $\mathrm{I}_{i d}$ List from respective $\mathrm{Table}_{i}$. All the retrieved $\mathrm{I}_{i d}$ Lists are stored in a temporary set. Finally, we count the number of occurrences (i.e., votes) of each palmprint ID and select the palmprints that are received more votes than a predefined threshold $\mathrm{T}$ as possible matches (i.e., candidate set).

Let $\mathrm{N}$ is the database size, a sequential search process takes $\mathrm{O}(\mathrm{N})$ time to identify a query. On the other hand, this technique compares the query with only $\mathrm{k}$ representatives and hence require $\mathrm{O}(\mathrm{k})$ time where $\mathrm{k}<<\mathrm{N}$.

\subsection{Representatives}

In this approach, selection of representatives is an important concern. The representative palmprints must be different from one another and should reflect the qualities of all classes ofusers in the database. In this work, we use a clustering technique to choose the representatives. Clustering partitions all user palmprints into set of groups such that palmprints in same group are equal or similar to each other whereas palmprints in different groups are dissimilar. As each group has similar palmprints, we select one palmprint from the group as its representative. Finally, the representative set contains palmprints that are from different classes of users.

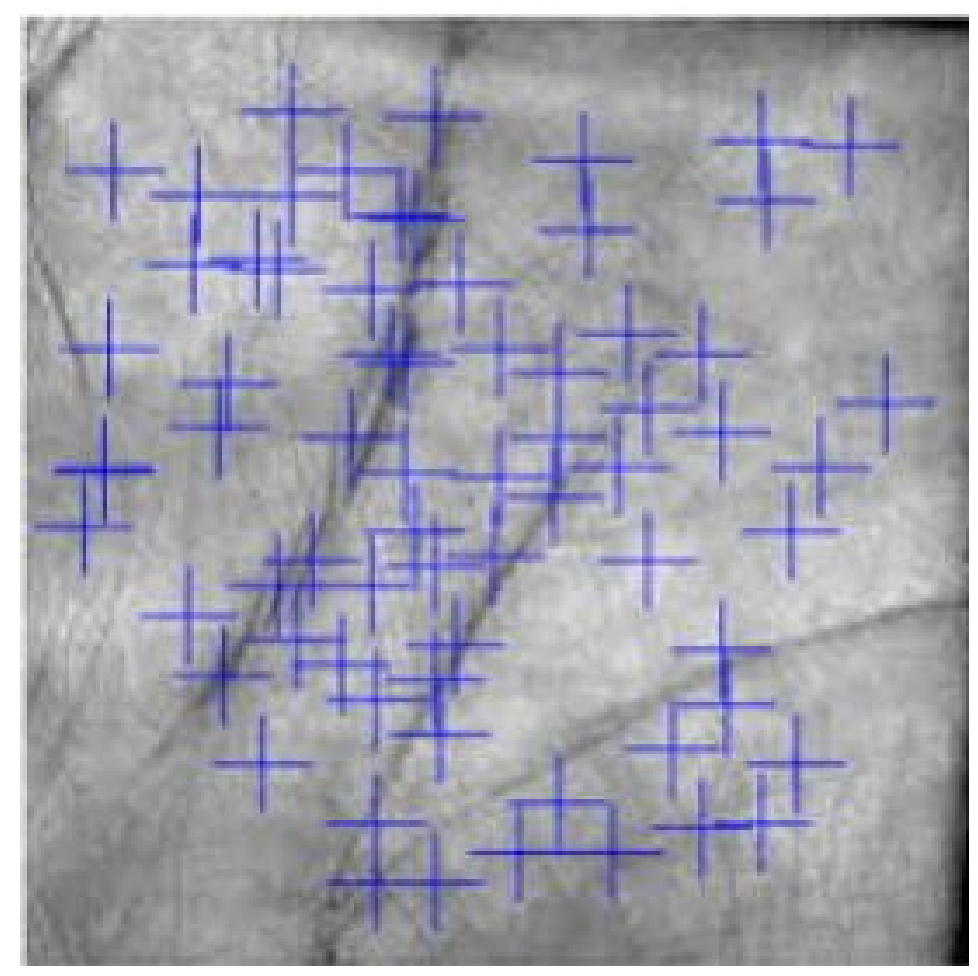

Fig 1. Sample palmprint image and its extracted SURF features

In this approach, an adaptive clustering called Leader algorithm ${ }^{(32-34)}$ is used for representative selection. The static clustering approach such as k-means algorithm has a serious limitation that, it has to re-cluster the database for every new enrollment which is a time consuming process and hence not suitable for a real time application. Further, the number of clusters 
also fixed. On the other hand, with Leader algorithm the new enrollments can be done dynamically and without affecting the existing system.

The Leader algorithm randomly chooses one of the palmprint as starting Leader and scans the database images one by one, and selects set of Leaders as cluster representatives. Every time, if a new palmprint to to make entry, it is assigned to the most similar cluster if exist. Otherwise, a new cluster is created and this palmprint becomes the Leader of that cluster.

\section{Experiments}

Experiments are conducted based on benchmark PolyU palmprint database ${ }^{(35)}$. This database contains 7752 palmprints of 386 different users approximately 20 palmprints per user. We have chosen randomly 10 palmprints per user for training and leftout things for testing. We segmented the palmprints into $151 \times 151$ pixels ${ }^{(36)}$. Figure 2 show a sample palmprint image and its extracted SURF features.

\subsection{Performance measures}

We evaluated the proposed system performance using three measures: Hit Rate (HR), Miss Rate (MR) and Penetration Rate (PR). HR shows the percentage of test palmprints to which the genuine palmprint is found in the candidate set.

$$
H R=\left(\frac{X}{M}\right) \times 100 \%
$$

where $\mathrm{X}$ represents the query palmprints count to which the genuine match is found in the retrieved candidate set and $\mathrm{M}$ is the count of palmprints tested.

MR represents the percentage of test palmprints to which the genuine match is not found in the candidate set.

$$
M R=100-H R
$$

PR describes the average size of candidate set generated for a query palmprint by the indexing technique.

$$
P R=\left(\frac{1}{M} \sum_{i=1}^{M} \frac{\left|L_{i}\right|}{N}\right) \times 100 \%
$$

where $\mathrm{L}_{i}$ represents candidate set size for $\mathrm{i}^{\text {th }}$ test palmprint, $\mathrm{N}$ is the total training palmprints taken and $\mathrm{M}$ shows the testing palmprints count.

\subsection{Selection of parameters}

The experimental results in this solution are involved with various parameters. In this proposed work, mainly the parameters with respect to Miss Rate and Penetration Rate have been considered. By considering threshold value the MR and PR parameters has been analysed. This section validates the optimal value for these parameters for which the system achieves its better performance. First, we validate the optimal value for the number of representatives. Next, we validate the selection rules for the representative images.

\subsubsection{Number of representatives}

The number of representatives i.e., k plays a primary role on the system performance. The low number of images may not represent the entire database. In contrast, very large number of images may leads to redundancy in the representative set. An optimal value should be selected so that the system achieves its better performance. An experiment is conducted by varying the number of representatives. As shown in Figure 3, the MR and PR of the system values decrease with k. Further, it can be seen that for the number of representatives greater than 130, the decrement in MR and PR is not that much significant. Hence, the optimal value for $\mathrm{k}$ is chosen as 130 .

\subsubsection{Selection rule for representatives}

The representative's selection rule is also an important concern of the system performance. We experimented with three different selection rules: randomly selected $\mathrm{k}$ images, first $\mathrm{k}$ images and Leader clustering ( Figure 3). It can be observed that clustering performs well than the other approaches. In other way, MR and PR of the system are low which shows the efficacy of the Leader 
algorithm for the representative's selection. Leader algorithm is a incremental clustering algorithm generally used to cluster large data sets. This algorithm is order dependent and may form different clusters based on the order the data set is provided to the algorithm.

\subsection{Results}

Once the optimal parameter values are chosen, we conducted an experiment by varying the threshold value from 0 to 100 and recorded the MR and PR values for the proposed approach ( Figure 5 ). It can be observed that there exist a trade-off between the PR and MR. The PR decreases with the threshold whereas MR increases.

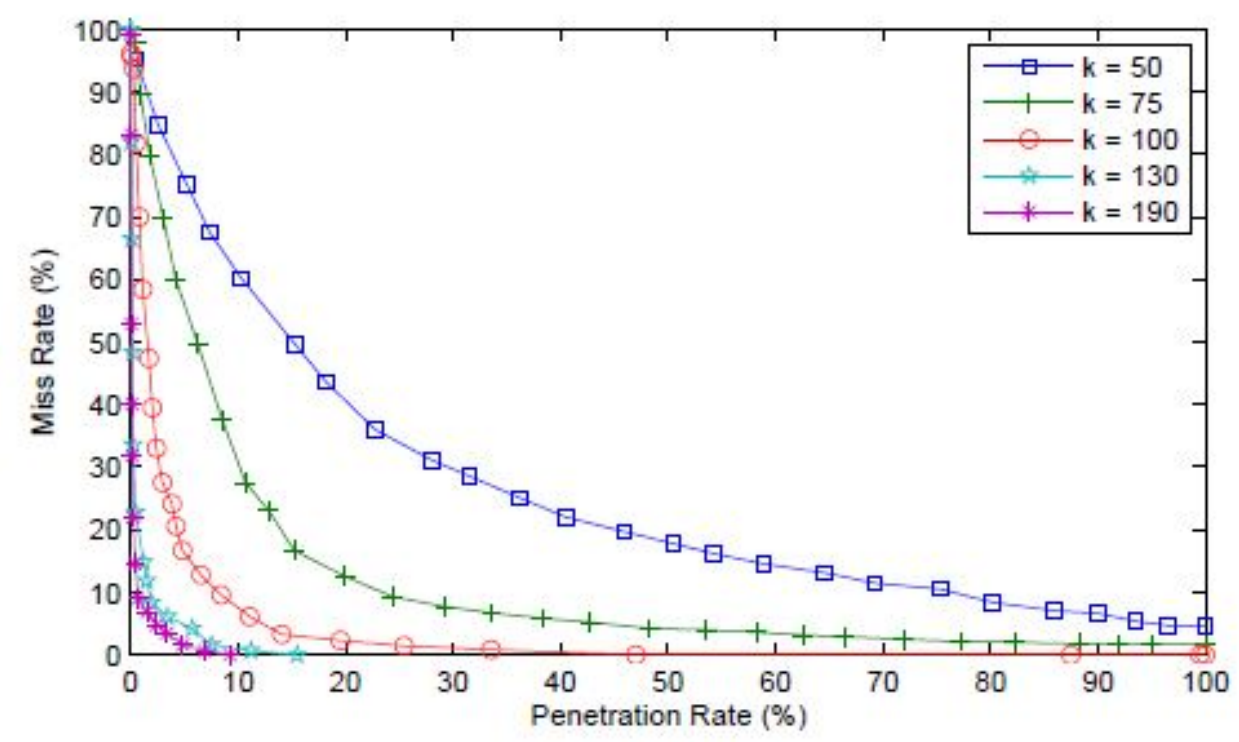

Fig 2. Indexing performance with the increasing representative set size $(\mathrm{k})$

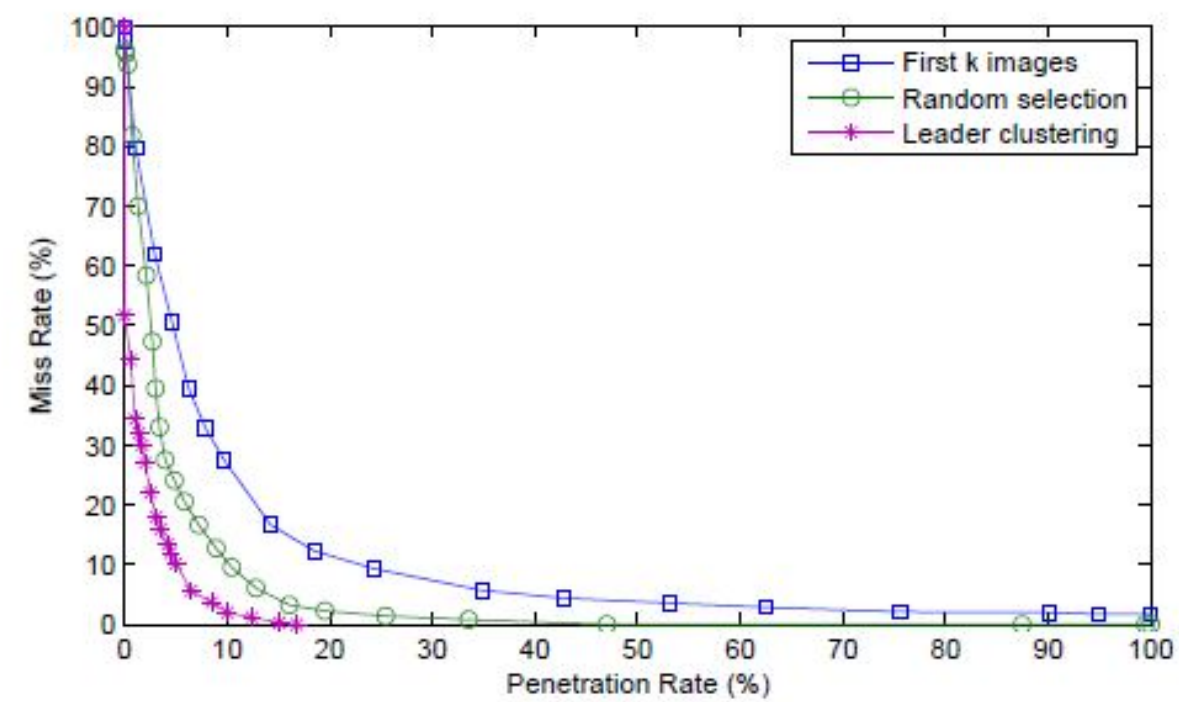

Fig 3. Indexing performance with different selection rules 
Hence we have to choose an optimum threshold value where the system achieves its better performance. But the optimum threshold value depends on the application where we are using this system. For a critical application like boarder security, identifying accuracy (i.e., genuine identification) should be high (i.e., HR is $100 \%$ and MR is $0 \%$ ). Hence for this type of applications, the optimum threshold value is chosen as threshold where the system can get MR is $0 \%$. On the other side, for general applications both PR and MR should be less. Based on different threshold value the MR and PR parameters have been analysed in Figure 4 .

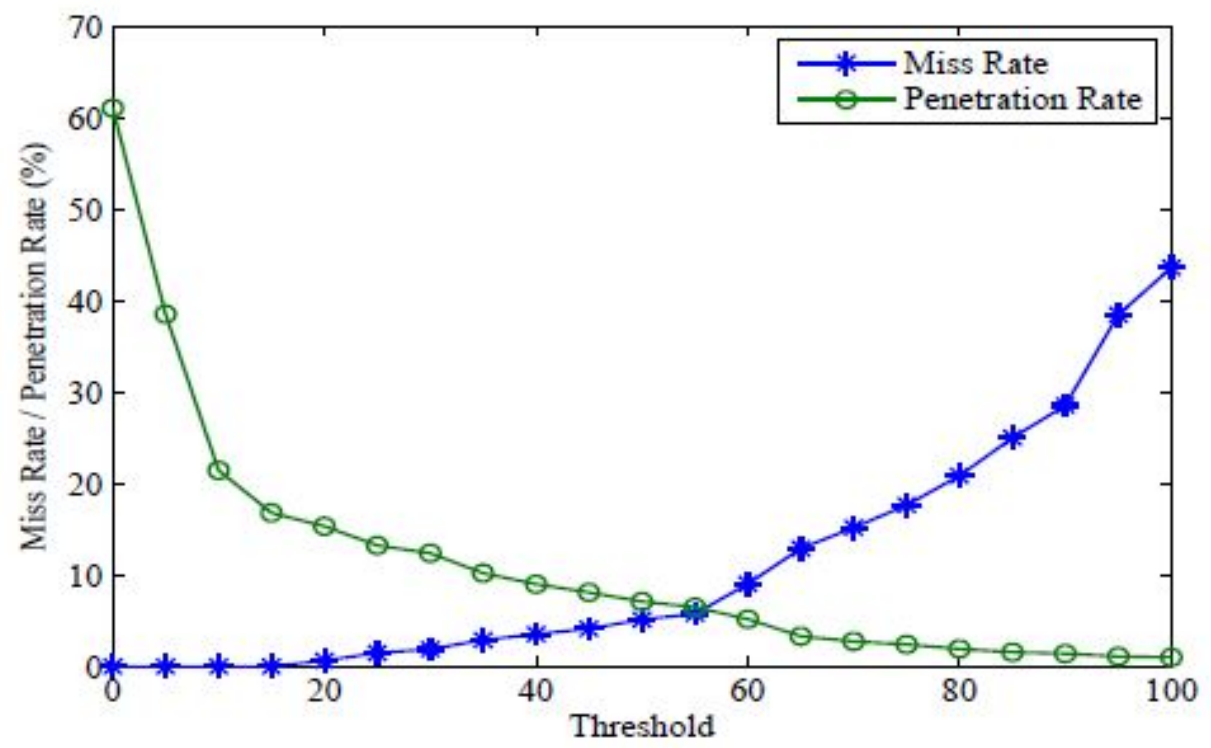

Fig 4. $P R$ and $M R$ of the system at different thresholds

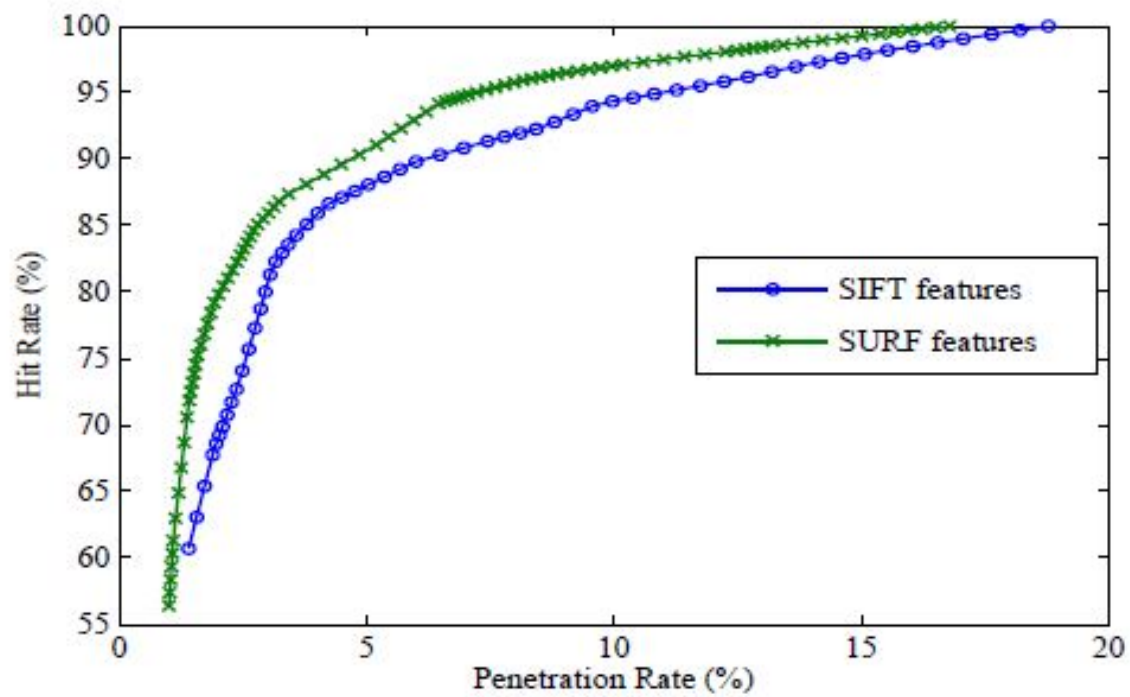

Fig 5. Performance comparison of the system with SIFT and SURF features

Hence for these applications, an optimum threshold is selected as the threshold where the two curves (i:e., $P R=M R$ ) intersect. It can be observed from Table 2 and Table 3 , that $\mathrm{MR}=0 \%$, the $\mathrm{PR}$ is $16.46 \%$. In other terms, proposed approach searches only $16.46 \%$ of the database to identify a query with $100 \%$ accurate. Further, at MR=PR our approach searches only $6.48 \%$ and 
identifies the query with $93.51 \%$ accuracy.

Table 2. The PR (\%) where $\mathrm{PR}=\mathrm{MR}$ and $\mathrm{MR}=0 \%$ of the proposed solution

\begin{tabular}{ll}
\hline & $\mathrm{PR} \%$ \\
\hline $\mathrm{MR}=\mathrm{PR}$ & $6.48 \%$ \\
$\mathrm{MR}=0 \%$ & $16.46 \%$ \\
\hline
\end{tabular}

Further, we also compare our approach with SIFT (Scale Invariant Feature Transform) features which we are used in our earlier work ${ }^{(27)}$. It can be observed from Figure 5 that the SURF features perform better than SIFT features.

Table 3. HR (\%) achieved using different techniques for 45 PolyU of PR (\%) of the system at maximum

\begin{tabular}{lll}
\hline Approach & HR & PR \\
\hline Proposed & $100 \%$ & $16.46 \%$ \\
$(14)$ & $98.28 \%$ & - \\
$(4)$ & $100 \%$ & $31.89 \%$ \\
\hline
\end{tabular}

\subsection{Comparison with other approaches}

We compare the proposed approach with ${ }^{(14)}$ technique which is also based on match scores and ${ }^{(4)}$ geometric hashing based method. In ${ }^{(14)}$ approach uses a vector estimated file to store the similar and matched scores. They have choses 170 images for the representative set and achieve only a maximum of $98.18 \% \mathrm{HR}$, for PolyU database. On the other side, our proposed solution used one thirty reference images and obtained $100 \%$ HR. In ${ }^{(4)}$ authors retrieved the key properties from the biometric images and it has been used the geometric hashing ${ }^{(37)}$ for indexing. But, the authors could gain a PR of $31.89 \%$ for PolyU database and shown the performance of various approaches.

\subsection{Effectiveness for new enrolments}

To enrol or register a new palmprint $\mathrm{x}$ to the existing system, we first compute its index keys against each representative and make an entry into the respective look up tables (see Section 3.1). Next, $\mathrm{x}$ is assigned to a closest cluster using its similarity score, if exists. Else, a new cluster is created and $\mathrm{x}$ is selected as its Leader. Further, a new look up table is created corresponding to this new representative and all palm prints are enrolled into it. This approach without effecting existing entries creates entry to the new users.

\section{Concluding remarks}

In this study, an efficient indexing technique is proposed for palm prints using SURF features and similarity scores. The defined look up table reduced the search time of the system by arranging the palm prints in sorted order. The use of Leader clustering for representative selection allows enrolling or creating the new users efficiently and without affecting the existing records. The retrieval time of the proposed approach is only $\mathrm{O}(\mathrm{k})$, where $\mathrm{k}$ is the number of representatives. On the other hand side, a linear search method ${ }^{(12)}$ requires $\mathrm{O}(\mathrm{N})$, here $\mathrm{N}$ is the database size and $\mathrm{k}$ 《 $\mathrm{N}$. The proposed could experiment on small database which contains of 7,753 images. This solution may able to show $100 \%$ accuracy when large databases considered. As a future work huge database can be considered using advanced techniques like BigData with suitable tools.

\section{References}

1) Maltoni D, Maio D, Jain AK, Prabhakar S. Handbook of Fingerprint Recognition. 2nd ed. and others, editor;Springer Publishing Company. 2009.

2) Mhatre A, Palla S, Chikkerur S, Govindaraju V. Efficient search and retrieval in biometric databases. In: and others, editor. Symposium SPIE Defense and Security. 2005;p. 265-273.

3) Boro R, Roy SD. Fast and robust projective matching for fingerprints using geo-metric hashing. In: and others, editor. Proceedings of the Fourth Indian Conference on Computer Vision, Graphics \& Image Processing. 2004;p. 681-688.

4) Badrinath GS, Gupta P, Mehrotra H. Score level fusion of voting strategy of geometric hashing and SURF for an efficient palmprint-based identification. J Real-Time Image Proc. 2013;8:265-284. Available from: https://doi.org/10.1007/s11554-011-0229-2.

5) Germain RS, Califano A, Colville S. Fingerprint matching using transformation parameter clustering. IEEE Computational Science and Engineering. 1997;4(4):42-49. Available from: https://dx.doi.org/10.1109/99.641608.

6) Ross A, Mukherjee R. Augmenting ridge curves with minutiae triplets for fingerprint indexing. In: and others, editor. Proc. SPIE. 2007. 
7) Bebis G, Deaconu T, Georgiopoulos M. Fingerprint identification using delaunay triangulation. In: and others, editor. International Conference on Information Intelligence and Systems. 1999;p. 452-459. Available from: https://doi.org/10.1109/ICIIS.1999.810315.

8) Gago-Alonso A, Hernández-Palancar J, Rodríguez-Reina E, Muñoz-Briseño A. Indexing and retrieving in fingerprint databases under structural distortions. Expert Systems with Applications. 2013;40(8):2858-2871. Available from: https://dx.doi.org/10.1016/j.eswa.2012.12.004.

9) Kavati I, Chenna V, Prasad MVNK, Bhagvati C. Classification of extended de-launay triangulation for fingerprint indexing. In: and others, editor. 8th Asia Modelling Symposium (AMS). IEEE. 2014;p. 153-158. Available from: https://doi.org/10.1109/AMS.2014.38.

10) Iloanusi NO. Fusion of finger types for fingerprint indexing using minutiae quadruplets. Pattern Recognition Letters. 2014;38(0):8-14. Available from: https://dx.doi.org/10.1016/j.patrec.2013.10.019.

11) Maeda T, Matsushita M, Sasakawa K, DBLP. Characteristics of the Identification Algorithm Using a Matching Score Matrix. In: and others, editor. Biometric Authentication, First International Conference, ICBA 2004, Hong Kong. 2004;p. 330-336.

12) Gyaourova A, Ross A. Index Codes for Multibiometric Pattern Retrieval. IEEE Transactions on Information Forensics and Security. 2012;7(2):518-529. Available from: https://dx.doi.org/10.1109/tifs.2011.2172429.

13) Kavati I, Prasad MVNK, Bhagvati C. An efficient coding method for indexing hand-based biometric databases. In: and others, editor. International Conference on Artificial Intelligence and Evolutionary Algorithms in Engineering Systems. Springer. 2015;p. 723-731. Available from: https://doi.org/10. 1007/978-81-322-2135-7_76.

14) Paliwal A, Jayaraman U, Gupta P. A score based indexing scheme for palmprint databases. In: and others, editor. International Conference on Image Processing. 2010;p. 2377-2380.

15) Kavati I, Prasad MVNK, Bhagvati C. A new indexing method for biometric databases using match scores and decision level fusion. In: and others, editor. International Conference on Ad-vanced Computing, Networking and Informatics;vol. 1. Springer. 2014;p. 493-500.

16) Paul M, Karsh RK, Talukdar FA. Image Hashing based on Shape Context and Speeded Up Robust Features (SURF). In: Computational and Technology Management (ICACTM). 2019;p. 464-468. Available from: https://doi.org/10.1109/ICACTM.2019.8776713.

17) Ansari S. A Review on SIFT and SURF for Underwater Image Feature Detection and Matching. In: 2019 IEEE International Conference on Electrical, Computer and Communication Technologies (ICECCT). 2019;p. 1-4. Available from: https://doi.org/10.1109/ICECCT.2019.8869489.

18) Hao J, Sen Y, Huang X. Based on Surf Feature Extraction and Insulator Damage Identification for Capsule Networks. In: 2019 International Conference on Computer Network, Electronic and Automation (ICCNEA). 2019;p. 301-306. Available from: https://doi.org/10.1109/ICCNEA.2019.00064.

19) Genovese A, Piuri V, Plataniotis KN, Scotti F. PalmNet: Gabor-PCA Convolutional Networks for Touchless Palmprint Recognition. IEEE Transactions on Information Forensics and Security. 2019;14(12):3160-3174. Available from: https://dx.doi.org/10.1109/tifs.2019.2911165.

20) Li X, Yang J, Ma J. Large Scale Category-Structured Image Retrieval for Object Identification Through Supervised Learning of CNN and SURF-Based Matching. IEEE Access. 2020;8:57796-57809.

21) Matkowski WM, Chai T, Kong AWK. Palmprint Recognition in Uncontrolled and Uncooperative Environment. IEEE Transactions on Information Forensics and Security. 2020;15:1601-1615. Available from: https://dx.doi.org/10.1109/tifs.2019.2945183.

22) Zhang L, Cheng Z, Shen Y, Wang D. Palmprint and Palmvein Recognition Based on DCNN and A New Large-Scale Contactless Palmvein Dataset. . Symmetry. 2018;10(4). Available from: https://doi.org/10.3390/sym10040078.

23) Ungureanu SA, Salahuddin S, Corcoran P, arXiv:2003 00737 [cs CV]. Towards Unconstrained Palmprint Recognition on Consumer Devices: a Literature Review. 2020. Available from: https://doi.org/10.1109/ACCESS.2020.2992219.

24) Fei L, Lu G, Jia W, Teng S, Zhang D. Feature Extraction Methods for Palmprint Recognition: A Survey and Evaluation. IEEE Transactions on Systems, Man, and Cybernetics: Systems. 2019;49(2):346-363. Available from: https://dx.doi.org/10.1109/tsmc.2018.2795609.

25) Wang J, Zhang X, Gong W, Xu X. A Summary of Palmprint Recognition Technology. In: 2019 4th International Conference on Control, Robotics and Cybernetics (CRC). 2019;p. 91-97. Available from: https://doi.org/10.1109/CRC.2019.00027.

26) Bay H, Tuytelaars T, Gool LV. SURF: Speeded Up Robust Features. In: Leonardis A, Bischof H, Pinz A, editors. Computer Vision - ECCV 2006. ECCV;vol. 3951. Springer. 2006. Available from: https://doi.org/https://doi.org/10.1007/11744023_32.

27) Kavati I, Prasad MVNK, Bhagvati C. Palmprint retrieval based on match scores and decision-level fusion. International Conference on Advances in Computing, Commu-nications and Informatics. 2015;p. 1591-1595. Available from: https://doi.org/10.1109/ICACCI.2015.7275840.

28) Khan SA, Iftikhar F, Akram U. Geometry Augmented SURF with Modified Sobel for Improved Affine Invariance in Image Matching. In: 2019 International Conference on Robotics and Automation in Industry (ICRAI). 2019;p. 1-6. Available from: https://doi.org/10.1109/ICRAI47710.2019.8967349.

29) Akhyar RM, Tjandrasa H. Image Stitching Development By Combining SIFT Detector And SURF Descriptor For Aerial View Images. In: 2019 12th International Conference on Information \& Communication Technology and System (ICTS). 2019;p. 209-214. Available from: https://doi.org/10.1109/ ICTS.2019.8850941.

30) Ordóñez A, Heras DB, Argüello F. Surf-Based Registration for Hyperspectral Images. In: IGARSS 2019 - 2019 IEEE International Geoscience and Remote Sensing Symposium. 2019;p. 63-66. Available from: https://doi.org/10.1109/IGARSS.2019.8900462.

31) Liu J, Bu F. Improved RANSAC features image-matching method based on SURF. The Journal of Engineering. 2019;2019(23):9118-9122. Available from: https://dx.doi.org/10.1049/joe.2018.9198.

32) Jain AK, Murty MN, Flynn PJ. Data clustering. ACM Computing Surveys (CSUR). 1999;31(3):264-323. Available from: https://dx.doi.org/10.1145/331499. 331504.

33) Vijaya P, Murty MN, Subramanian D. LeadersâASsubleaders:, An efficient hier-archical clustering algorithm for large data sets. Pattern Recognition Letters. 2004;25(4):505-513.

34) Kavati I, Prasad MV, Bhagvati C. A clustering-based indexing approach for bio-metric databases using decision-level fusion. International Journal of Biometrics. 2017;9(1):17-43.

35) The polyu palmprint database. . Available from: http://www.comp.polyu.edu.hk/biometrics.

36) Lin CL, Chuang CT, Fan KC. Palmprint verification using hierarchical decomposition. Pattern Recognition. 2005;38(12):2639-2652. Available from: https://dx.doi.org/10.1016/j.patcog.2005.04.001.

37) Wolfson HJ, Rigoutsos I. Geometric hashing: an overview. IEEE Computational Science and Engineering. 1997;4(4):10-21. Available from: https: //dx.doi.org/10.1109/99.641604. 